\title{
Critical reflection on the role of theater nurses in a multidisciplinary team for perioperative care in China
}

Discussion

Xiao Xiao, She-Ning Zhu ${ }^{\mathrm{b}, * *}$

${ }^{a}$ Obstetric Department, Shenzhen Maternity and Child Healthcare Hospital, Shenzhen, Guangdong 518028, China

${ }^{b}$ Nursing Administration Department, Shenzhen Maternity and Child Healthcare Hospital, Shenzhen, Guangdong 518028, China

Received: 21 October 2018; Accepted: 22 December 2018; Published: 20 March 2019

Abstract: 0 bjective: The aim of this article is to reflect on the role of theater nurses in a multidisciplinary team, understand the factors that have influenced theater nurses' practice, and improve the authors' clinical practice ultimately.

Methods: The author used Smyth's model to guide the process of reflection on the practice issue. Critical reflection, critical emancipatory theory, reflexivity, and critical social theory were used to help the author analyze the factors that have affected theater nurses' practice in the organization.

Results: There are gaps between the espoused and enacted theories. A theater nurse's practice is determined by multiple factors, such as political, structural, social, historical, cultural issues, and so on. The hierarchy of the health context could hinder possible changes in theater nurses' practice. To better understand our practice and implement transformation, we should shape a supportive environment, bear in mind the practice motto of "patient-centered" care, and improve our knowledge and reflection skills.

Conclusions: Reflection plays a significant role in the advancing of practice among theater nurses and needs to be combined with clinical practice. To provide the best service of care to perioperative patients, a theater nurse should have an insightful understanding of the factors that have influenced her/his behaviors historically, socially, and culturally. By improving their critical reflection skills, practitioners could gain knowledge from experience.

Keywords: multidisciplinary team $\bullet$ Smyth's reflective framework $\bullet$ reflexivity $\bullet$ critical reflection $\bullet$ critical emancipatory theory $\bullet$ critical social theory $\bullet$ task-oriented $\bullet$ person-centered care

(c) Shanxi Medical Periodical Press.

\section{Introduction}

Reflection on practice is regarded as crucial to responsible professional practice, ${ }^{1}$ since there are always gaps between formal theory and actual practice. ${ }^{2}$ The focus of critical reflection is thinking in new ways and moving toward more critical practice. ${ }^{3}$ It is an antecedent of critical action, because it helps professionals to understand the social and structural contexts and constraints that have impeded their personal power. ${ }^{1}$ As a result, it allows health professionals to challenge and change the hegemonic structures in the organization. ${ }^{4}$ Additionally, critical reflection emphasizes the intuitive and artistic aspects of practitioners' practice and understands their emotions in certain circumstances. ${ }^{1}$ Thus, critical reflection could help

How to cite this article: Xiao X, Zhu SN. Critical reflection on the role of theater nurses in a multidisciplinary team on perioperative care in China. Front Nurs. 2019; 1: xx-xx. 
practitioners enrich their knowledge, reduce the gap, and detect the actual theory that underpinned their practice. ${ }^{2}$ In other words, reflection is a way to improve practice.

\section{Methods}

In this article, the author chose critical reflection, critical emancipatory theory, reflexivity, and critical social theory to underpin the process of reflection. Through the process of critical reflection, the author will be able to critically articulate the practice issues from the first person perspective. Guided by these theories, the author will interpret the role and social obligations of theater nurses in the multidisciplinary team on perioperative care for patients; examine the power relationships in the context the author is embedded in; critically analyze the personal, sociocultural, historical, and political factors that have influenced theater nurses' clinical practice. Ultimately, the author might change the actions, feel free of takenfor granted assumptions, transform future practice, and have a better understanding of clinical practice. ${ }^{5}$ Smyth's ${ }^{6}$ reflective framework will be tailored to assist the process of reflection and an example scenario will be utilized to highlight the practice issues discussed in the paper. At the end of reflection, the author's understanding can be maximized, which will facilitate the uncovering of the nature of the oppressions that inhibit theater nurses.

\section{Results}

\subsection{Describe}

I have been working as a theater nurse for 8 years since I graduated from the university. As a theater nurse, part of my work is visiting postoperative patients the first day after surgery. Each time, I have to be in a hurry to visit all the patients because of my busy timetable. Once, I engaged with a lady; she was in her first day after salpingectomy for ectopic pregnancy. She kept asking me so many questions that I supposed should be answered by doctors and nurses in the ward. I could answer those questions, but I did not know how to give her a perfect answer. Therefore, I suggested that she could ask nurses in the wards and went away to visit the next patient. To avoid being questioned, I would finish the conversation with patients as soon as possible.

\subsection{Inform}

What do these mean to me? I found that I could do nothing for this because I was not sure about these questions. I felt upset, anxious, and stressed when I communicated with her and feared that she would repeat her questionnaire again. I knew that the woman was visibly upset and needed my care at that moment, and no response from me might add to her suffering. ${ }^{7}$ Maybe I should stop to comfort her and try to find the answers to her questions; however, other patients might also expect me to care for them and visit them first. ${ }^{7}$ I was ethically correct to respond to the woman, rather than "kick the ball" to another nurse or doctor as if I am interchangeable or incompetent. ${ }^{7}$

Actually, the voice from within told me that I should maintain my professional status quo; it seems as if I dare not tell patients that I could not answer their questions. ${ }^{7}$ I was fearful of my weakness and incompetence being seen by patients ${ }^{7}$; otherwise, they might distrust me and suspect whether I was a professional nurse. ${ }^{8}$ Therefore, I felt uncomfortable and guilty for hiding my anxiety and uncertainty. At the same time, I felt a sense of danger regarding my dignity that arose from a professional identity, ${ }^{8}$ though Van den Heever et al. ${ }^{7}$ mentioned that it is impossible for health providers to have all the answers.

On the other hand, I believe that communication between patients and nurses is very important. ${ }^{9}$ At some point, it seemed I was willing to answer their questions; however, sometimes, their questions were beyond my capability; I felt frustrated and embarrassed each time I was caught by endless questions. It seemed that I was "mopping up a mess"; my body urged me to visit the next patient as soon as possible and work as though on a "production line". I assumed task-oriented routines were effective ways to ensure that my work is completed; however, these might distract me from seeing and responding to patients' individuals needs. ${ }^{10}$ Sometimes, even though I could identify their needs, it seemed as if I was impeded by some power in the organization and the fragmented care and the wavelength set by the hegemony. ${ }^{10}$ My insight also told me that the woman might not be a self-disciplined person and I felt that I should keep away from those who are pregnant before marriage. Furthermore, it seemed that I was not well prepared for the postoperative visit. This was not the first time that I was being asked these questions: each time I refuse to answer these intermittent questions with the sound excuse that it is none of my business or that I have no time. Maybe it is just a way for me to "run away" from the task that I am not confident in.

\subsection{Confront}

I questioned myself "why am I acting like this"? Reflexivity claims that I should understand my practice issue in terms of broader institutionalized norms and how the context has influenced my practice. ${ }^{1}$ From the political stance, I knew I should care for patients and comfort them. ${ }^{11}$ I should act according to my duty and responsibilities defined by my professional standard. While I was not the women's nurse-in-charge nor did I have any form 
of ward authority, ${ }^{12}$ from my leader's perspective, my primary role should be ensuring that I visit all postoperative patients on time and provide quality care at the same time. ${ }^{13}$ Hence, I have to enhance my work efficiency and complete my work on time; otherwise, I might be criticized as possessing low work efficiency.

However, the reality in practice is that I am very busy with my work; the staffing and resources are limited; even in a well-developed health system, I still feel that my role was so overloaded that I had to squeeze the caring aspect. ${ }^{14}$ As a result, I felt that my working environment was very pressurized and demanding, and the great workload gave me little time to care for the patients' needs. ${ }^{8}$ Furthermore, inadequate training activities for perioperative nurses leave me ill-equipped with relevant knowledge and skills, apart from few opportunities for me to discuss my concerns. Thus, my frustration was increased when I could not meet the standard practice.$^{15}$ From this perspective, the postoperative visit itself might have problems.

Christie et al. ${ }^{16}$ said that it is difficult to demonstrate "person-centered" care in a busy health setting. There are multiple priorities in the culture of my hospital; both patient satisfaction and work efficiency are valued. ${ }^{17}$ There would always be conflicting priorities; it is difficult for me to balance the two. ${ }^{8}$ On the other hand, working extra hours is common within the health-care system without extra pay. As a nurse, I was blamed for bad work manners by doctors, family members, other nurses, and nurse managers during the process of meeting patients needs. ${ }^{8}$ Without positive feedback from anybody, my work behavior might lead to compassion fatigue toward my work. ${ }^{8}$ I think that leaders' expectations of individual, tailored care and pursuit of patients' satisfaction is not practical if the staff do not have the opportunity to create a caring moment. ${ }^{8}$ I had a keen desire to challenge and change the situation when I was at the bottom of the hierarchy system, and I found that I was powerless when confronting the hegemony. My frustration associated with the health system that I was a part of had negatively affected my inspiration toward work. ${ }^{18}$

In addition, my role in the multidisciplinary team shows that my obligation is associated with intraoperative nursing rather than postoperative advocacy. If I work across boundaries, it might lead to conflicts between multidisciplinary teams; ${ }^{19}$ thus, I would comfort myself that maybe it should not be my personal responsibility to care for postoperative patients. Sometimes, I have to give up some beliefs regarding what is best and find a middle path to train the whole team about what is right; this might add to my burden of moral distress. ${ }^{12}$

At some point, I recognized that my personality might also have affected my way of communicating with others. My past experience of failing to express myself effectively had always led to misunderstanding by others. I was a sensitive person; I valued how my actions were perceived, and how my actions would affect the state of my relationship with others. Consequently, I become wordless in front of people to avoid being misunderstood. Actually, I should have told the concerned doctor or nurse about the woman's concerns and reminded them to care for her more, or I could have returned to her later if I had time because effective nursing communication should take patients' feelings into consideration, listen to patients, and supply the information they need. ${ }^{20}$

Sometimes, maybe I just want to protect myself from being harmed when I withdraw from patients. ${ }^{8}$ । am fed up with the occupational violence reported by public media. Some patients and their relatives attacked nurses and doctors because of their dissatisfaction with the service they received. ${ }^{21}$ Currently, trust between health professionals and patients is poor; without mutual respect and trust, multidisciplinary collaboration would be frustrating and challenging. ${ }^{20}$

I noted that my value was also underpinned by my task-oriented work mode. When I am struggling with taskoriented work in the theater, I have no time to understand patients' feelings. ${ }^{8} \mathrm{I}$ am used to working with instruments and equipment, and I have forgotten my responsibility to care for patients and how to communicate with patients effectively. ${ }^{22}$ Sometimes, it seemed as if I had "lost my mind" and had become a machine that extends service. ${ }^{4}$ I felt that I had no time to stop and think, and being with a patient seemed like a premium. Maybe I have got accustomed to getting the work done and am suffering from compassion fatigue, so I try to avoid difficult questions and conversations. ${ }^{23}$ Therefore, an interruption of my work is often viewed as a nuisance. ${ }^{24}$

In Chinese culture, the public is infused with ideas of self-sacrifice for the benefit of patients. ${ }^{23}$ In a therapeutic relationship, patients are vulnerable compared with health professionals, who have powerful medical knowledge. ${ }^{8}$ The public does not care about the workload of nurses and the areas that they work in, but they only appreciate whether their experiences in the hospital are good or bad. Because the health service in China is not free, and most of our salary and welfare are generated from patients' payment for our services, ${ }^{23}$ the idea that they have paid money for our services is rooted in their mind. Medical staff should act according to their preference and they deserve quality care as expected, especially some "difficult patients" who could limit my capacity and add to my negative feelings. ${ }^{25}$

From the perspective of the public, as a health professional, I should be professional and knowledgeable. I could not make mistakes; I should practice perfectly, as if I should know everything. If my answer was to be wrong, patients would give me an "incompetent" label. ${ }^{9}$ 
Nevertheless, I think the public should not imagine that everything could be cured in hospitals and that professionals should know everything. ${ }^{26}$ I felt that the expectations from patients and public are too high in some way; consequently, health professionals experience too much pressure from these hopes. ${ }^{8}$ Nobody shows sympathy for health professionals.

In addition, the stereotype of "ashamed of unmarried pregnancy" has been deeply embedded in my mind. Influenced by Chinese traditional culture, these women would be judged by the public to be self-abandoned, and that they should be responsible for their own behaviors. ${ }^{27}$ Therefore, it is common for medical staff and public to discriminate against this group unconsciously. I got insight into my prejudices and understood that this might have inhibited me from caring for the woman with her specific needs. ${ }^{28}$ Guided by my assumptions, sometimes, I might show different working manners toward different people.

The critical social theory implies that better understanding of my behavior, my practice, and my beliefs should be based on the analysis of both historical and structural factors. ${ }^{29}$ I would deny the existence of my constrained values and beliefs and be unable to challenge the power relationships if I fail to interpret my historically constructed habits. ${ }^{6}$

Historically, the essence of nursing is caring for people, and it is my duty to promote patients' well-being. The Chinese nurses' code of conduct ${ }^{11}$ suggests that nurses are in a position to help patients through the following: promote better health; advocate healthy lifestyles to patients; comfort patients; and provide empathetic care and psychological support to patients. Nurses should maintain continuous learning to meet patients' and their family members' needs; at the same time, nurses should also work collaboratively with other professionals. Therefore, influenced by the ideology of the ethics of nursing that I had been taught at the university and my code of conduct in the context of the Chinese health system, I was in a position to care for patients. I know that I should provide "person-centered" care to patients, and it is good for patients. ${ }^{13}$

However, in the health organization, health workers are not appreciated by the local supervisors but instead are scrutinized to prevent them from making mistakes. ${ }^{30}$ Therefore, the relationship between colleagues might be tense. ${ }^{30}$ Lack of incentives, such as low salaries and few opportunities for promotion, might also contribute to my low motivation toward work. ${ }^{30}$ And I am not even concerned about my performance because of demotivation. ${ }^{30}$

In terms of postoperative care, there is no clarification about each role in a multidisciplinary team. ${ }^{12}$ My work and life experience tell that if I interfere with other disciplines' work, I might bring about conflicts between my colleagues and me. To sustain a good relationship with my colleagues, I have to clearly identify my role in my workplace. ${ }^{12}$ In the traditional medical model, illnesses, efficiency, standards, and the needs of health professionals are more valued. ${ }^{10}$ I was smitten by the assumption that I was more powerful than patients. ${ }^{10}$ Doctors have more authority than I did in a medical model, and nurses are perceived to be subordinates in the health-care system. ${ }^{31}$ Thus, even if I know that nurses might not practice properly, I should maintain silence rather than disclose their practice. ${ }^{28}$

The hierarchy in my work is so deeply embodied and embedded within the health context in China that it would be challenging for me to shrug off the tradition. Though multidisciplinary collaboration has been advocated, fear of change might be a constraint that might disrupt the possible benefits for patients and me.28 Therefore, identifying my place in a team and recognizing the barriers generated by the organizational structure might have hindered me from transforming and bringing about some possible changes in some way. ${ }^{28}$

\subsection{Reconstruct}

When reconstructing my practice issue, I could think in an alternative way. Maybe patients were anxious to know their situation, while doctors and nurses in the ward were busy, and I happened to come across at that moment. I should think from the patients' perspective, trust them, and understand their feelings rather than questioning their trustworthiness of me.

Improvement of knowledge and skills could enhance my confidence and competence to work in a "personcentered" way and help me recognize my limitations and barriers, which have hindered me from working differently. ${ }^{29}$ I could summarize the most frequent questions that patients care most and prepare for these questions or make an individual plan with the whole perioperative team. A briefing gets all team members to reach a commitment and provides a structure for collaborative planning. ${ }^{31}$ This communication then shapes a shared mental model of how that particular patient encounter will proceed. ${ }^{32}$ In addition, sharing knowledge within the multidisciplinary team is also crucial. ${ }^{33}$ Maybe I should learn some knowledge on postoperative care so that I can provide better services to patients. Alternatively, if each time I visit patients, I gather patients together, my work efficacy could be enhanced, and I might have more time to communicate with patients and pay more attention to holistic care. ${ }^{9}$

However, one of the premises of "person-centered" care is transforming the work environments. ${ }^{34}$ Vetter et al. ${ }^{35}$ stated that the complexity of science gives us a new understanding of health-care organizations, whereby hierarchy and the controlled model are obsolete. Emergent leadership could foster creativity and promote 
positive relationships and better patient care.$^{35} \mathrm{~A}$ blamefree working environment encourages health-care professionals to report and learn from each other's events in work without concerns. ${ }^{34}$ Working in a supportive environment facilitates health professionals exploring current practices critically, permits an equal opportunity to speak freely and discuss the difficult issues together, and find positive solutions. ${ }^{16}$ On the other hand, "person-centered" practice can be interpreted in different ways, and working efficiency, in a broad thinking, could be an alternative that benefits most patients.$^{10}$ In addition, there might be conflicts between evidence-based practice and "meeting individualized needs". ${ }^{10}$

Continuous care could enhance patients' postoperative experience, promote mutual familiarity, and reduce patient and family's anxiety consequently. ${ }^{35}$ Therefore, it is necessary to set standards for postoperative care. ${ }^{35}$ There is a need to shape a "person-centered" culture within my hospital, whereby I am equipped with interpersonal skills, shared values and beliefs in my team, adequate staffing, and accountable leadership to facilitate person-centeredness; ${ }^{35}$ then, I can work together with my other colleagues to provide better care to postoperative patients. This has been echoed by Cotter, ${ }^{4}$ who suggested that only if people reach a commitment and work together could they be deemed to possess freedom and power.

\section{Discussion}

Reflection could help health professionals learn from experience and, thus, develop and maintain competency through lifelong learning. ${ }^{36}$ Though there is no clear evidence to show the correlation between reflective practice and competency, reflection has become a mandatory component of professional practice. ${ }^{36}$ Each individual is used to defining themselves from the "habits of mind" or "their point of view", while critical reflection could emancipate oneself from externally imposed views and beliefs. ${ }^{37}$ In this article, I used reflection-on-action; retrospective reflection on the patient, as a result, I identified why I acted like that. As I become more "expert"

\section{References}

1. Fook J, Gardner F. Practising Critical Reflection: $A$ Resource Handbook. Berkshire: Open University Press. 2007.

2. Schon D. Educating the Reflective Practitioner. San Francisco, CA: Jossey-Bass. 1987.

3. Tretheway R, Taylor J, O'Hara L, Percival N. A missing ethical competency? A review of critical reflection in health promotion. Health Promot $J$ Austr. 2015;26:216-221. in my practice, I will develop my clinical reasoning and reflect in action and be able to change my practice then and there, rather than after the event.

The increasing demands of quality care from both patients and organizations often put nurses in dilemmas while defining their professional roles. ${ }^{8}$ In the future, I should enhance my critical reflection skills, keep reflecting on the day's clinical situations by writing a diary or a daily journal, and see a situation from multiple perspectives. ${ }^{38}$ I should allow myself to spare some time to stand back, since this will lead to better choices in my practice and work more effectively in the long run. ${ }^{38}$ However, seeking change does not depend on a single individual or organization but acts on multiple levels simultaneously. ${ }^{38}$ As an agent of change, I could implement a change in my individual behavior, while an organizational change should count on enough individual changes. ${ }^{38}$ Because "person-centered" care should count on all stakeholders working collaboratively through shared leadership, the first step should enhance the team members' engagement and ensure their commitment to a common goal, communicated effectively among team members. ${ }^{38}$

\section{Conclusions}

Reflection plays a significant role in the development and advancement of practice of theater nurses and needs to be combined with clinical practice. Each theater nurse aspires to provide the best service in terms of care to perioperative patients. ${ }^{36}$ However, to achieve this, a theater nurse should have an insightful understanding of the factors that have influenced her/his behaviors historically, socially, and culturally. Through the development of critical reflection skills, practitioners will gain knowledge from experience; change their behaviors, and know how to work in a team to provide better service to patients.

\section{Conflicts of interest}

All contributing authors declare no conflicts of interest.

4. Cotter R. Reflexive spaces of appearance: rethinking critical reflection in the workplace. Hum Resou Dev Int. 2014;17:459-474.

5. Taylor B. Reflective Practice for Healthcare Professionals: A Practical Guide. 3rd ed. New York: McGraw-Hill Education. 2010.

6. Smyth J. Developing and sustaining critical reflection in teacher education. J Teach Educ. 1989; 40:2-9. 
7. Van den Heever AE, Poggenpoel M, Myburgh $\mathrm{CPH}$. Nurses' perceptions of facilitating genuineness in a nurse-patient relationship. Health SA Gesondheid. 2015;20:109-117.

8. Morrison KB, Korol SA. Nurses' perceived and actual caregiving roles: identifying factors that can contribute to job satisfaction. J Clin Nurs. 2014;23:3468-3477.

9. West M, Eckert R, Steward K, Pasmore B. Developing Collective Leadership for Health Care. London: The King's Fund. 2014.

10. Christie J, Camp J. Critical reflection on the process of validation of a framework for person-centered practice. Int Pract Dev J. 2014;48:1-11.

11. Pan SS, Zhang JZ, Zhang XQ, Xie HZ, Li EC, Jia QA. Content interpretation of "ethical guidelines for nurses". Chin Med Ethics. 2014;27:468-470 (in Chinese).

12. Engel J, Prentice D. The ethics of interprofessional collaboration. Nursing Ethics. 2013;20:426-435.

13. Health Service Executive. Framework for Improving Quality in Our Health Service. Dublin: Health Service Executive. 2016.

14. Coetzee SK, Klopper HC. Compassion fatigue within nursing practice: a concept analysis. Nurs Health Sci. 2010;12:235-243.

15. Mellinger E. Knowing "something is not right" is beyond intuition: development of a clinical algorithm to enhance surveillance and assist nurses to organize and communicate clinical findings. J Clin Nurs. 2015;24:832-843.

16. Christie J, Camp J, Cocozza K, et al. Finding the hidden heart of healthcare: the development of a framework to evidence person-centered practice. Int Pract Dev J. 2012;2:1-22.

17. Bergh AL, Friberg F, Persson E, Dahlborg-Lyckhage E. Perpetuating 'new public management' at the expense of nurses' patient education: a discourse analysis. Nurs Inq. 2014;22:190-201.

18. Wickford J, Rosberg S. From idealistic helper to enterprising learner: critical reflections on personal development through experiences from Afghanistan. Physiother Theory Pract. 2012;28:283-291.

19. McNamara MS, Fealy GM, Casey M, et al. Boundary matters: clinical leadership and the distinctive disciplinary contribution of nursing to multidisciplinary care. J Clin Nurs. 2011;20:3502-3512.

20. Hu YY, Arriaga AF, Peyre SE, Corso KA, Roth EM, Greenberg CC. Deconstructing intraoperative communication failures. J Surg Res. 2012;177:37-42.

21. Hahn S, Müller M, Needham I, Dassen T, Kok G, Halfens RJ. Factors associated with patient and visitor violence experienced by nurses in general hospitals in Switzerland: a cross-sectional survey. $J$ Clin Nurs. 2010;19:3535-3546.

22. Liaw SY. A review of educational strategies to improve nurses' roles in recognizing and responding to deteriorating patients. Int Nurs Rev. 2011;58:296-303.

23. Chen $M$, Wang $L$, Chen W, Zhang $L$, Jiang $H$, Mao W. Does economic incentive matter for rational use of medicine? China's experience from the essential medicines program. Pharmacoeconomics. 2014;32:245-255.

24. McGibbon E, Peter E, Gallop R. An institutional ethnography of nurses' stress. Qual Health Res. 2010;20:1353-1378.

25. Dawber C. Reflective practice groups for nurses: a consultation liaison psychiatry nursing initiative: part 2 - the evaluation. Int $J$ Ment Health Nurs. 2013;22:241-248.

26. Sabo BM. Compassion fatigue and nursing work: can we accurately capture the consequences of caring work? Int J Nurs Pract. 2006;12:136-142.

27. Huang JJ. Reflection on the phenomenon of unmarried pregnancy among Chinese juveniles. Chin $J$ Hum Sexual. 2007;16:36-37 (in Chinese).

28. Asselin ME, Schwartz-Barcott D, Osterman PA. Exploring reflection as a process embedded in experienced nurses' practice: a qualitative study. $J$ Adv Nurs. 2013;69:905-914.

29. Crowe MT, O'Malley J. Teaching critical reflection skills for advanced mental health nursing practice: a deconstructive-reconstructive approach. J Adv Nurs. 2006;56:79-87.

30. Mbindyo P, Gilson L, Blaauw D, English M. Contextual influences on health worker motivation in district hospitals in Kenya. Implement Sci. 2009;4:43.

31. Salhani D, Coulter I. The politics of interprofessional working and the struggle for professional autonomy in nursing. Soc Sci Med. 2009;68:1221-1228.

32. Award SS, Fagan SP, Bellows C, et al. Bridging the communication gap in the operating room with medical team training. Am J Surg. 2005;190:770-774.

33. Körner M, Lippenberger C, Becker S, et al. Knowledge integration, teamwork and performance in health care. $J$ Health Organ Manage. 2016;30:227-243.

34. Ammouri AA, Tailakh AK, Muliira JK, Geethakrishnan R, Al Kindi SN. Patient safety culture among nurses. Int Nurs Rev. 2014;62:102-110.

35. Vetter TR, Goeddel LA, Boudreaux AM, Hunt TR, Jones KA, Pittet JF. The perioperative surgical home: how can it make the case so everyone wins? BMC Anesthesiol. 2013;13:6. 
36. Paterson C, Chapman J. Enhancing skills of critical reflection to evidence learning in professional practice. Phys Ther Sport. 2013;14: 133-138.
37. Mezirow J. Transformative learning: theory to practice. New Dir Adult Contin Educ. 1997;74:5-12.

38. Gardner F. Creating a climate for change. Int $J$ Knowledge Culture Change Manage. 2007;6:73-80. 